\title{
Redes neuronales artificiales y modelo de regresión lineal múltiple: nuevas alternativas para mejorar la estimación de gasto energético total en jóvenes
} universitarios

\author{
Artificial Neural Networks and Multiple linear regression model: \\ New alternatives for enhacing the prediction of total energy \\ expenditure in university students
}

\author{
FABIOLA MORENO-CRUZ* y JORGE SÁNCHEZ-HERRERA
}

Departamento de Nutrición, Universidad del Valle de México (UVM), Campus Lomas Verdes, Instituto de Estudios Superiores en Administración Pública (IESAP), Ciudad de México, México

\section{RESUMEN}

La Encuesta Nacional de Salud y Nutrición de Medio Camino 2016, señala que el sobrepeso y la obesidad son las alteraciones del estado nutricio con mayor prevalencia en la población mexicana, esto se traduce en un alto riesgo de desarrollar patologías crónico-degenerativas a futuro. Una oportuna estimación del gasto energético total (GET) deriva en intervenciones preventivas en la población de jóvenes. Utilizando la metodología de minería de datos con dos máquinas de aprendizaje, redes neuronales artificiales (RNA) y el modelo de regresión lineal múltiple (MRLM), donde a partir de ocho variables explicatorias (sexo, edad, peso, talla, masa grasa, masa libre de grasa, agua corporal total y actividad física) se estimó el GET. Los resultados obtenidos mediante ambos métodos presentan un valor promedio absoluto de desviación ( $\left.\varepsilon \_a\right)$ de 121 y 116 respectivamente, menor al calculado por fórmulas de estimación $\left(\varepsilon \_a=558\right)$ tomando como referencia el cálculo de este a partir de bioimpedancia eléctrica. Se concluye que ambas máquinas de aprendizaje, las RNA y el MRLM, obtuvieron promedios de desviaciones absolutas menores a las obtenidas con las ecuaciones de Harris-Benedict y Mifflin-St. Jeor. Hoy en día el uso de la ciencia de datos representa un área de oportunidad para lograr impactos positivos en el sistema de salud en México.

Palabras clave: Minería de datos. Redes neuronales artificiales. Regresión lineal múltiple. Gasto energético total. Ciencia de datos y máquinas de aprendizaje.

\begin{abstract}
The National Mid-Way Health and Nutrition Survey 2016 indicates that overweight and obesity are the alterations of the nutritional status with the highest prevalence in the Mexican population, this translates into a high risk of developing chronic degenerative pathologies in the future. A timely estimate of Total Energy Expenditure (GET) results in preventive interventions in the youth population. Using the data mining methodology with two learning machines, Artificial Neural Networks (RNA) and Multiple Linear Regression Model (MRLM), where from eight explanatory variables (gender, age, weight, height, fat mass, fat free mass, total body water and physical activity) the GET was estimated. The results obtained by both methods have an absolute average deviation value (z_a) of 121 and 116 respectively, lower than that calculated by estimation formulas $\left(\varepsilon_{\_} a=558\right)$, taking as reference the calculation of this from electrical bioimpedance. It is concluded that both, RNA and MRLM learning machines, obtained averages of absolute deviations smaller than those obtained with Harris-Benedict and Mifflin-St. Jeor equations. Today the use of data science represents an area of opportunity to achieve positive impacts on the health system in Mexico.
\end{abstract}

Key words: Data mining. Artificial neural networks. Multiple linear regression. Total energy expenditure. Data science and learning machines.
Correspondencia:

*Fabiola Moreno-Cruz

E-mail: fa_cruz28@hotmail.com
Fecha de recepción: 09-01-2019

Fecha de aceptación: 18-12-2019

DOI: 10.24875/RME.19001887
Disponible en internet: $25-02-2020$

Rev Mex Endocrinol Metab Nutr. 2020;7:29-36

2462-4144 / ๔ 2019 Sociedad Mexicana de Nutricion y Endocrinologia, AC. Publicado por Permanyer. Este es un artículo open access bajo la licencia CC BY-NC-ND (http://creativecommons.org/licenses/by-nc-nd/4.0/). 


\section{INTRODUCCIÓN}

Hoy en día el sobrepeso y la obesidad son las alteraciones del estado nutricio con mayor prevalencia en la población mexicana. De acuerdo con la Encuesta Nacional de Salud y Nutrición de Medio Camino 2016, la prevalencia combinada de sobrepeso y obesidad en adultos de 20 años o más es del $71.2 \%{ }^{1}$. Los agentes etiológicos para el desarrollo de estas patologías son múltiples, entre ellos factores ambientales, alimenticios y de estilo de vida, por mencionar algunos. Por tanto, se asume que la adopción de una dieta correcta y de estilos de vida saludable pueden fungir como aspectos preventivos. De acuerdo con la Organización Mundial de la Salud, el gasto energético total (GET) es el nivel de energía necesario para mantener el equilibrio entre el consumo de alimentos y el gasto energético de un individuo sano, debiendo hacer ajustes en diferentes estados fisiológicos o patológicos ${ }^{2}$. La diferencia entre la ingesta y el gasto de energía, a menudo denominada balance energético es de gran interés, debido a su relación directa con la ganancia o pérdida a largo plazo de tejido adiposo. Por esta razón, han surgido preguntas acerca de si la ingesta y el gasto energético se pueden medir adecuadamente con la finalidad de poder realizar un cálculo calórico más exacto que permita mantener en equilibrio el balance energético, contribuyendo a un mejoramiento en el control de masa corporal y energía².

La determinación del GET considera el gasto energético en reposo (GER), la actividad física y el efecto termogénico de los alimentos (ETA). Existen varias características fisiológicas que hacen que el GER varíe de persona a persona, las principales son: sexo, edad, peso, talla, masa grasa, masa libre de grasa (MLG), agua corporal total y actividad física ${ }^{2}$. El nivel de actividad física se describe como la proporción entre el GET y el GER y se usa para determinar la cantidad e intensidad de la actividad física habitual de un individuo; es decir, la cantidad de energía que está siendo utilizada para este fin $^{2}$.

El ETA se refiere al aumento del gasto energético producido después del consumo de alimentos, y corresponde a la energía necesaria para la digestión, absorción, transporte, metabolismo y almacenamiento de los macronutrimentos, equivalente al $10 \%$ de la energía contenida en los alimentos de una dieta mixta ${ }^{3}$.

EI GER puede ser medible a partir de diferentes métodos. La evidencia señala que la calorimetría directa se considera el estándar de oro para la medición. Este método mide la cantidad de calor producida por el organismo y se realiza en cámaras herméticas con paredes aislantes, en donde se registra el calor almacenado y el perdido por radiación, convección y evaporación. Se precisa un mínimo de seis horas para estabilizar el sistema; es un método complejo y difícil de realizar en la práctica, por tanto, su uso ha sido de carácter investigativo o para valorar métodos indirectos ${ }^{2}$. Sin embargo, otro determinante del GER es la composición corporal, especialmente la MLG. Por ello existen diferentes métodos para determinarlo: antropometría, agua doblemente marcada, densitometría, absorciometría con rayos $\mathrm{X}$ de doble energía, impedancia eléctrica, etc. No obstante, y debido al alto costo que representa la medición del GER se realiza habitualmente por medio de ecuaciones predictivas validadas, las de HarrisBenedict y Mifflin-St. Jeor entre las más utilizadas ${ }^{3}$.

Hoy en día se dispone de equipos de medición que tienen incorporado en su software las ecuaciones para determinar el GER, lo que se considera una ventaja, ya que además de contener las ecuaciones predictivas, toman en cuenta diversos compartimentos corporales que influyen en este. La bioimpedancia eléctrica es un método que estima los compartimentos corporales y tiene su fundamento en la resistencia al paso de la corriente alterna ${ }^{3}$. El tejido magro es altamente conductor debido a la gran cantidad de agua y electrolitos que contiene, por tanto, ofrece baja resistencia; por el contrario, la grasa, la piel y la masa ósea son bajos conductores y por ende altamente resistentes ${ }^{2}$. Este método ha adquirido gran uso en los últimos años, ya que no es invasivo, presenta una rápida aplicación, es seguro y relativamente económico en comparación con los métodos anteriormente mencionados; sin embargo, en México aún no se cuenta en todas las unidades médicas con el equipo y personal adecuado para realizar una correcta valoración del estado 


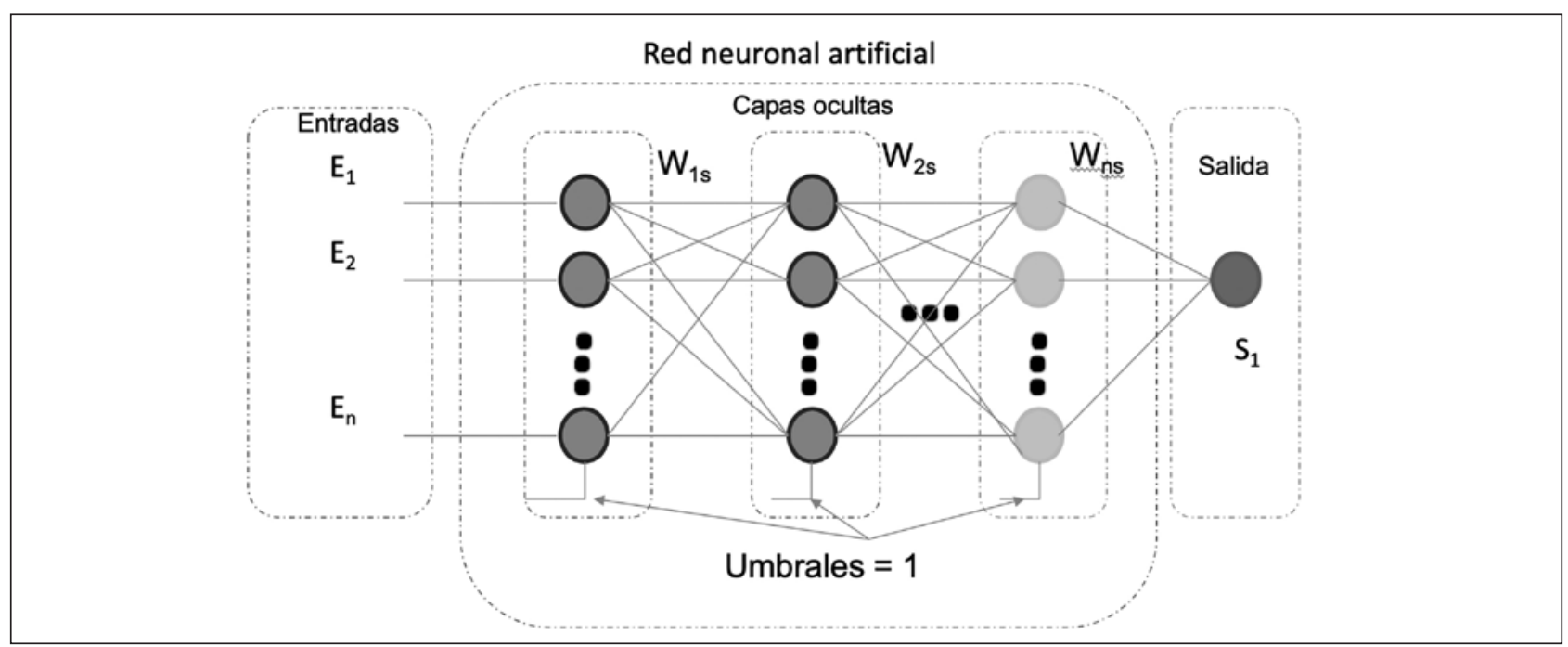

Figura 1. Red neuronal artificial.

nutricio, lo que dificulta el cálculo del GET y hace, por ende, más compleja la prescripción dietética ${ }^{4}$. La nutrición representa la piedra angular del tratamiento para el sobrepeso y la obesidad, y la adecuada estimación del GET forma parte crucial para un tratamiento y resultados exitosos.

Los sistemas de salud en México cuentan con una gran cantidad de datos que pueden ser utilizados para resolver los desafíos en materia de salud. Una forma de lograrlo es mediante el uso de ciencia de datos (minería de datos, big data e inteligencia artificial) ${ }^{5}$.

\section{Redes neuronales artificiales}

La minería de datos es el proceso de descubrimiento de conocimiento y está basada en modelos matemáticos, entre otros los conocidos como máquinas de aprendizaje (redes neuronales artificiales [RNA] y el modelo de regresión lineal múltiple [MRLM]).

Las RNA son ampliamente utilizadas para estimar valores de variables dependientes en modelos explicatorios, así como para la clasificación de datos ${ }^{6}$. Extrapolan con un alto grado de simplificación el concepto de operación de las redes neuronales biológicas a algoritmos computacionales; operan con múltiples capas de neuronas artificiales, interconectadas entre sí
(Fig. 1). Resumiendo, el algoritmo consiste en reducir el error cuadrático entre el dato esperado y el dato calculado, a saber:

$$
E=(\text { Esperado }- \text { Calculado })^{2}
$$

- La RNA está compuesta de múltiples capas (entrada, ocultas y salida), cada una de las cuales tiene distinto número de neuronas.

- Cada neurona tiene asociada:

- Entradas de datos.

- Valores numéricos, conocidos como pesos $\left(\mathrm{W}_{\mathrm{s}}\right)$, en igual cantidad al número de entradas; estos emulan la función de sinapsis.

- Un valor conocido como bias, que hace la función de umbral de activación de la neurona.

- Función de propagación, que se define como:

$$
F_{p}=\sum E_{i} * W_{j i}
$$

- Función de activación (lineal o no lineal), que transforma la función de propagación:

$$
S_{i}=F_{a}\left(F_{p}\right)
$$

- Ejecuta múltiples interacciones conocidas como épocas. En cada una de estas realiza un recorrido hacia adelante para que, a partir de los datos de entrada y las transformaciones realizadas, estime el valor de salida; adicionalmente, realiza un recorrido hacia atrás, utilizando el método matemático 
conocido como gradiente descendente para el ajuste de pesos:

$$
W_{\text {new }}=W_{\text {old }}-\eta \frac{\partial E}{\partial w}
$$

- La capa de salida puede tener una o más neuronas, dependiendo del problema que aborda; para el caso de este trabajo, tiene solo una.

$$
S_{f}=\sum F_{a}\left(F_{p}\right)
$$

- La finalización de ejecución de la RNA se da cuando termina el número de épocas u otro criterio de conclusión.

$$
W_{\text {new }}=W_{\text {old }}-\eta \frac{\partial E}{\partial w}
$$

\section{Modelo de regresión lineal múltiple}

El método de regresión lineal múltiple se utiliza para predecir una cantidad o número, es decir, dado un conjunto de datos de la forma:

$$
D=\{(x(i, j), y(j)): i, j=1 \ldots n\}
$$

donde $x(i, j) \in R^{n} y(j) \in R$.

El fundamento matemático se encuentra en el álgebra lineal, al calcular la proyección de un vector y (j) sobre un subespacio vectorial formado por los vectores $X_{n}$.

Vectorialmente se presenta como:

$$
\beta=\left(X^{T} X\right)^{-1} X^{T} Y
$$

Es decir, se encuentra la ecuación lineal:

$$
y_{i}=w \cdot x=w_{0}+w_{1} x_{1}+\ldots+w_{n} x_{n}
$$

que minimiza la suma del error cuadrático en el valor de $y_{i}$ y el valor estimado de $\widehat{y}_{l}$, es decir:

$$
\operatorname{MinE}=\sum\left(y_{i}-\widehat{y}_{l}\right)^{2}
$$

\section{METODOLOGÍA}

El trabajo corresponde a una investigación aplicada, que busca herramientas y alternativas para apoyar la toma de decisiones en la formulación de políticas públicas sobre salud, siendo la población de estudio la de jóvenes universitarios del Estado de México.

\section{Diseño de la investigación}

Los datos pertenecen a 64 pacientes que asistieron a los consultorios de nutrición de la Universidad del Valle de México (UVM), campus Lomas Verdes. Cada individuo expresó su conformidad para participar en la investigación.

\section{Criterios de inclusión}

- Tener un rango de edad de 18 a 40 años.

- Ser estudiante de la UVM.

- No tener ninguna patología diagnosticada.

- No consumir ningún fármaco.

- No haber ingerido alimentos ni bebidas 4 horas antes de la medición.

\section{Criterios de exclusión}

- Tener más de 40 años.

- No pertenecer a la comunidad estudiantil de la UVM.

- Tener alguna patología diagnosticada.

- Consumir algún medicamento.

- Haber consumido alimentos o bebidas durante el periodo de restricción.

\section{Criterios de eliminación \\ - Retirada del consentimiento informado.}

\section{Recogida de información}

La recogida de información se realizó mediante entrevistas personales. El instrumento consistió en una historia clínica-nutriológica de 25 ítems, entre los que se encuentran: sexo, edad, peso, talla, antecedentes patológicos, actividad física y hábitos de alimentación, tomando como base la Norma Oficial Mexicana NOM-004-SSA3-2012, del Expediente Clínico, así como las medidas correspondientes a la 
Tabla 1. Gasto energético total obtenido mediante la fórmula

Cálculo del GET utilizando las ecuaciones de Harris-Benedict y Mifflin-St. Jeor

\begin{tabular}{lcccccccc}
\hline Edad & Peso & Talla & $\begin{array}{c}\text { Porcentaje } \\
\text { MLG }\end{array}$ & $\begin{array}{c}\text { Agua } \\
\text { corporal }\end{array}$ & GER & GET & Calculado & $\begin{array}{c}\text { Error absoluto } \\
\text { abs(calculado - GET) }\end{array}$ \\
\hline 21 & 58.8 & 163.0 & 36.9 & 29.0 & 1,248 & 1,922 & 1,431 & 491 \\
\hline 22 & 63.9 & 166.8 & 40.9 & 32.1 & 1,292 & 1,705 & 1,482 & 223 \\
\hline 21 & 57.1 & 164.8 & 45.3 & 35.0 & 1,426 & 2,196 & 1,418 & 778 \\
\hline 40 & 78.8 & 167.3 & 49.8 & 39.1 & 1,401 & 1,849 & 1,541 & 1.104 \\
\hline 23 & 98.9 & 178.2 & 73.2 & 56.8 & 1,907 & 2,937 & 1,833 & 438 \\
\hline 21 & 100.6 & 172.9 & 62.1 & 48.8 & 1,711 & 2,259 & 1,821 & 565 \\
\hline Promedio de error absoluto & & & & & & & & \\
\hline
\end{tabular}

GER: gasto energético en reposo; GET: gasto energético total; MLG: masa libre de grasa.

bioimpedancia eléctrica. La toma de medidas antropométricas (peso, talla, porcentaje de grasa, masa grasa, MLG) y datos complementarios se realizó utilizando análisis de composición corporal por medio de bioimpedancia eléctrica (Gaia Kiko 359 PLUS ${ }^{\circledR}$, Jawon Medical). Los pacientes se seleccionaron de acuerdo con los criterios de inclusión establecidos. Se utilizó el software de minería de datos $\mathrm{KNIME}^{\circledR}$ versión 4.0.2.; los atributos seleccionados se transformaron en formato de valores separados por comas (formato CSV), para ser utilizables por la aplicación informática.

Los datos fueron particionados en dos grupos: $90 \%$ para entrenamiento del modelo y $10 \%$ para pruebas y contraste entre resultados.

\section{RESULTADOS OBTENIDOS}

Se incluyeron 64 estudiantes que asistieron a los consultorios de nutrición de la UVM, campus Lomas Verdes, que cumplieron con los criterios de inclusión del estudio, con edad media de 21.5 años; el $60.93 \%$ correspondían al sexo femenino y el $39.06 \%$ al masculino. Los pacientes presentaron un promedio de índice de masa corporal (IMC) de $25.21 \mathrm{~kg} / \mathrm{m}^{2}$, lo que los sitúa en un diagnóstico nutricio de sobrepeso.

El conjunto de datos fue particionado, para utilizar 57 registros en el entrenamiento de los algoritmos de RNA y MRLM, y los siete complementarios para realizar la evaluación de resultados.
Cálculo del gasto energético total utilizando las ecuaciones de HarrisBenedict y Mifflin-St. Jeor

Mediante el uso de las ecuaciones de Harris-Benedict y Mifflin-St. Jeor se obtuvo un valor promedio absoluto de desviación con respecto al GET calculado por bioimpedancia eléctrica, igual a 558 puntos (Tabla 1).

\section{Cálculo del gasto energético total utilizando redes neuronales artificiales}

El modelo de RNA se construyó utilizando la herramienta KNIME ${ }^{\circledR}$ (Fig. 2). Los datos empleados fueron: sexo, edad, peso, talla, MLG, agua corporal total y actividad física. Para evitar sesgos producidos por diferentes escalas de los datos, estos fueron normalizados en la escala mín.-máx.: 0-1, para ambos casos (entrenamiento y predicción), aplicando función inversa (desnormalización para la presentación de resultados).

La RNA obtuvo un valor promedio absoluto de desviación con respecto al GET calculado por bioimpedancia eléctrica igual a 121 puntos (Tabla 2).

\section{Cálculo del gasto energético total utilizando el modelo de regresión lineal múltiple}

El MRLM se construyó utilizando la herramienta KNIME ${ }^{\circledR}$ (Fig. 3). Los datos empleados fueron: edad, peso, talla, MLG, agua corporal total y actividad física. 


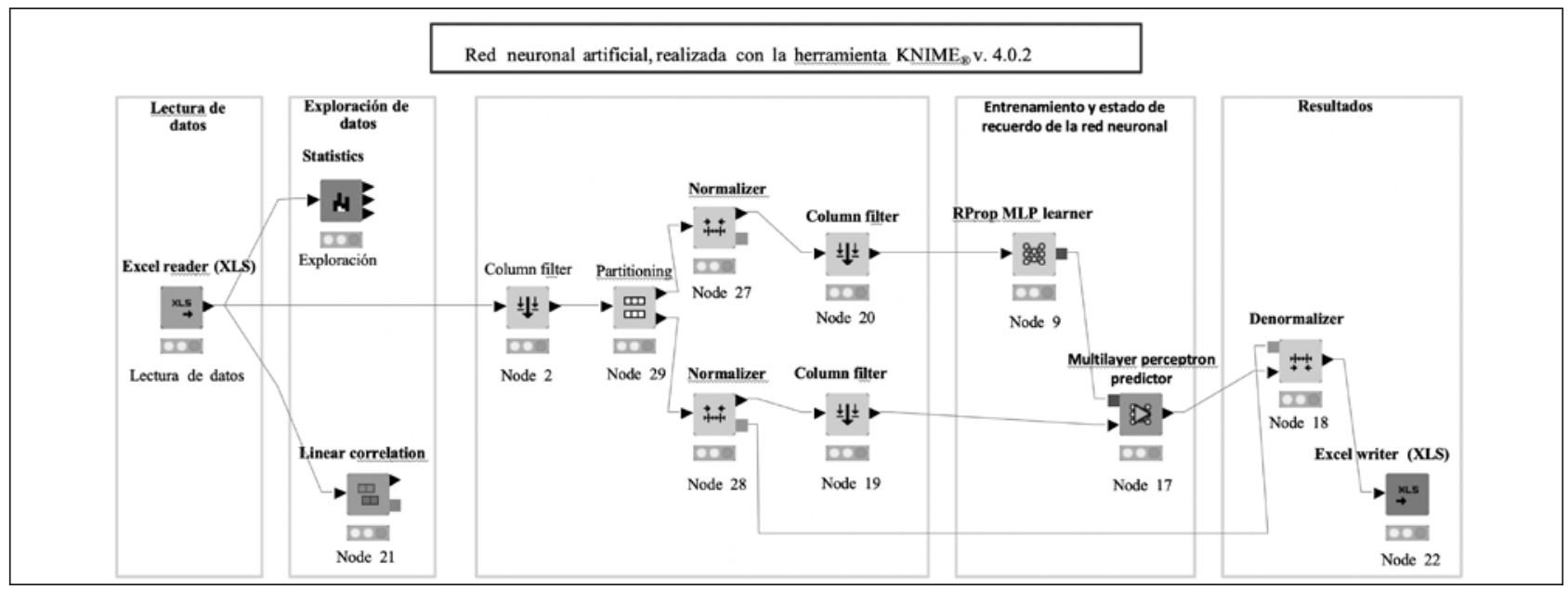

Figura 2. Redes neuronales artificiales para obtener el gasto energético total.

Tabla 2. Gasto energético total obtenido mediante redes neuronales artificiales

\begin{tabular}{|c|c|c|c|c|c|c|c|c|}
\hline \multicolumn{9}{|c|}{ Cálculo del GET utilizando RNA } \\
\hline Edad & Peso & Talla & $\begin{array}{l}\text { Porcentaje } \\
\text { MLG }\end{array}$ & $\begin{array}{l}\text { Agua } \\
\text { corporal }\end{array}$ & GER & GET & $\begin{array}{l}\text { Neuronal } \\
\text { obtenido }\end{array}$ & $\begin{array}{c}\text { Error absoluto } \\
\text { abs(calculado - GET) }\end{array}$ \\
\hline 21 & 58.8 & 163.0 & 36.9 & 29.0 & 1,248 & 1,922 & 1,709 & 213 \\
\hline 22 & 63.9 & 166.8 & 40.9 & 32.1 & 1,292 & 1,705 & 1,950 & 245 \\
\hline 21 & 57.1 & 164.8 & 45.3 & 35.0 & 1,426 & 2,196 & 2,213 & 17 \\
\hline 40 & 78.8 & 167.3 & 49.8 & 39.1 & 1,401 & 1,849 & 1,843 & 6 \\
\hline 23 & 98.9 & 178.2 & 73.2 & 56.8 & 1,907 & 2,937 & 2,937 & 0 \\
\hline 27 & 100.6 & 172.9 & 62.1 & 48.8 & 1,711 & 2,259 & 2,407 & 148 \\
\hline 21 & 77.5 & 159.5 & 48.8 & 38.3 & 1,408 & 2,168 & 1,947 & 221 \\
\hline \multicolumn{8}{|c|}{ Promedio de error absoluto } & 121 \\
\hline
\end{tabular}

GER: gasto energético en reposo; GET: gasto energético total; MLG: masa libre de grasa; RNA: redes neuronales artificiales.

Siguiendo el criterio aplicado en las RNA, los datos fueron normalizados en la escala mín.-máx.: 0-1, para ambos casos (entrenamiento y predicción), aplicando función inversa (desnormalización para la presentación de resultados).

EL MRLM obtuvo un valor promedio absoluto de desviación con respecto al GET calculado por bioimpedancia eléctrica igual a 116 puntos (Tabla 3).

\section{DISCUSIÓN}

La ciencia de datos está revolucionando la sociedad moderna. Los avances hoy en día nos han llevado al uso de alternativas tecnológicas para la optimización de procesos. La inteligencia artificial se ha convertido en una disciplina científica enfocada a proveer soluciones de la vida cotidiana, incluyendo los principales problemas de salud pública que aquejan al mundo. Existen diversos estudios en el ámbito de la medicina que han utilizado estos modelos matemáticos; en la bibliografía el uso más frecuente es como auxiliar de diagnóstico clínico. En Finlandia se desarrolló un abordaje de inmunodeficiencias primarias mediante inteligencia artificial comparado con un grupo de expertos de amplia experiencia, los resultados preliminares arrojaron 469 casos correctamente clasificados, lo que supone un $66 \%$ de efectividad del modelo ${ }^{7}$.

Seixas, et al. utilizaron RNA para diagnosticar tuberculosis pleural solo con parámetros clínicos y el estado de infección por el virus de la inmunodeficiencia humana, 
Tabla 3. Gasto energético total obtenido mediante modelo de regresión lineal múltiple

\begin{tabular}{lcccccccc}
\hline Edad & Peso & Talla & $\begin{array}{c}\text { Porcentaje } \\
\text { MLG }\end{array}$ & $\begin{array}{c}\text { Agua } \\
\text { corporal }\end{array}$ & GER & GET & Calculado & $\begin{array}{c}\text { Error absoluto } \\
\text { abs(calculado - GET) }\end{array}$ \\
\hline 21 & 58.8 & 163.0 & 36.9 & 29.0 & 1,248 & 1,922 & 1,871 & 51 \\
\hline 22 & 63.9 & 166.8 & 40.9 & 32.1 & 1,292 & 1,705 & 1,923 & 218 \\
\hline 21 & 57.1 & 164.8 & 45.3 & 35.0 & 1,426 & 2,196 & 2,241 & 45 \\
\hline 40 & 78.8 & 167.3 & 49.8 & 39.1 & 1,401 & 1,849 & 1,919 & 70 \\
\hline 23 & 98.9 & 178.2 & 73.2 & 56.8 & 1,907 & 2,937 & 2,678 & 259 \\
\hline 21 & 100.6 & 172.9 & 62.1 & 48.8 & 1,711 & 2,259 & 2,200 & 59 \\
\hline Promedio de error absoluto & & & & 1,408 & 2,168 & 2,055 & 113 \\
\hline
\end{tabular}

GER: gasto energético en reposo; GET: gasto energético total; MLG: masa libre de grasa; MRLM: modelo de regresión lineal múltiple.

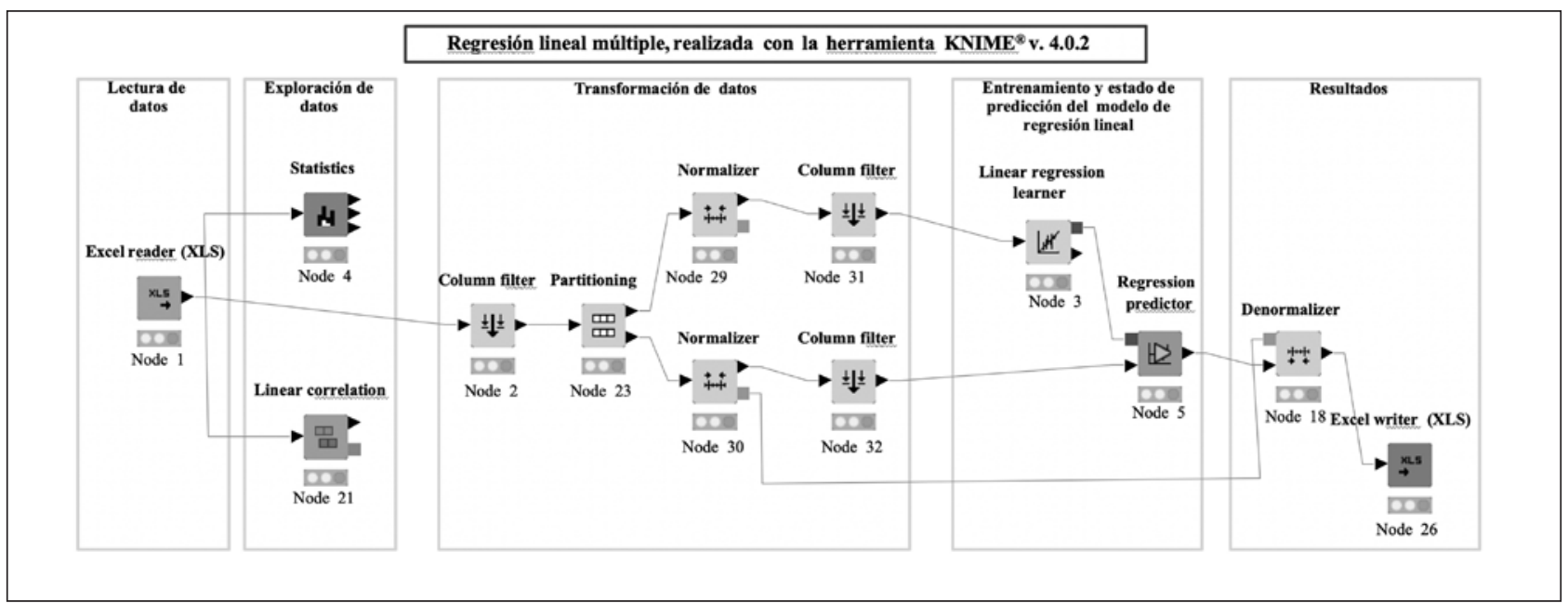

Figura 3. Modelo de regresión lineal múltiple para obtener el gasto energético total.

a partir de una base de adiestramiento de 137 pacientes. El desempeño fue superior a todas las otras pruebas diagnósticas, como biopsia, cultivo, ensayo por inmunoabsorción ligado a enzimas (ELISA), proteína C reactiva (PCR), etc., alcanzando más del $90 \%$ de exactitud $^{8}$. En México en el año 2011 especialistas en el IBM Watson trabajaron en colaboración con médicos del Instituto Nacional de Ciencias Médicas y Nutrición Salvador Zubirán, para identificar si los tratamientos médicos que habían elegido los expertos del hospital coincidían con el tratamiento recomendado por Watson, que había sido entrenado por el Memorial Sloan Kettering Cancer Center. Se seleccionaron 100 casos de cáncer de mama, pulmón, colon y recto, y se obtuvo un $46 \%$ de coincidencia en el tratamiento para cáncer de mama, un 50\% en cáncer de pulmón, un 79\% en cáncer de colón y un $100 \%$ en cáncer de recto. Las causas probables por las que no se alineara el tratamiento sugerido con el de los médicos expertos fue el costo, disponibilidad del medicamento y guías de tratamiento internacionales europeas más que estadounidenses ${ }^{9}$.

En materia de nutrición existen pocos estudios que utilicen este modelo. En Bolivia se hizo un estudio del gasto energético corporal en 30 pacientes de ambos sexos de 13 a 30 años. En los resultados se pudo observar que sí existía correlación con el modelo de comparación utilizado (antropometría); el porcentaje de acierto del sistema de expertos (inteligencia artificial) en comparación al modelo fue de un $97 \%$, por lo que se puede afirmar que es una herramienta efectiva y de apoyo al experto nutricionista. Comparando este estudio con el nuestro, podemos observar que la inteligencia artificial 
funge como una herramienta que coadyuva al diagnóstico y tratamiento de patologías relacionadas con la nutrición, logrando de esta manera el diseño de un plan de alimentación basado en las necesidades y características individuales de la población ${ }^{10,11}$.

\section{CONCLUSIONES Y RECOMENDACIONES}

Los resultados de ambos métodos, RNA y MRLM, obtuvieron promedios de desviaciones absolutas menores a las obtenidas con las ecuaciones de Harris-Benedict y Mifflin-St. Jeor, al pasar de 558 puntos a 121 y 116 respectivamente.

Las ecuaciones de estimación toman como únicas variables sexo, peso, talla y edad, tendiendo a sobreestimar o subestimar el GET, en contraste con los modelos empleados, que utilizaron ocho atributos (sexo, edad, peso, talla, masa grasa, MLG, agua corporal total y actividad física).

Hoy en día el uso de la ciencia de datos (minería de datos, big data e inteligencia artificial) se está incorporando al área de la salud; sin embargo, hay pocos trabajos relacionados con el área nutricia, lo que representa un área de oportunidad para la realización de futuros estudios, con la finalidad de lograr un impacto positivo en el sistema de salud en México, que coadyuve a los profesionales del área de la salud en la prevención y tratamiento nutricional de la creciente epidemia de sobrepeso y obesidad.

\section{RECONOCIMIENTOS}

A la UVM, campus Lomas Verdes, por permitir la realización del estudio a la población universitaria.

\section{FINANCIAMIENTO}

La presente investigación no ha recibido ninguna beca específica de agencias de los sectores público, comercial, o sin ánimo de lucro.
CONFLICTO DE INTERESES

Los autores declaran no tener conflicto de intereses.

\section{RESPONSABILIDADES ÉTICAS}

Protección de personas y animales. Los autores declaran que para esta investigación no se han realizado experimentos en seres humanos ni en animales.

Confidencialidad de los datos. Los autores declaran que han seguido los protocolos de su centro de trabajo sobre la publicación de datos de pacientes.

Derecho a la privacidad y consentimiento informado. Los autores han obtenido el consentimiento informado de los pacientes y/o sujetos referidos en el artículo. Este documento obra en poder del autor de correspondencia.

\section{BIBLIOGRAFÍA}

1. Encuesta Nacional de Salud y Nutrición de Medio Camino 2016. Informe final de resultados [Internet]. México: Secretaría de Salud, Instituto Nacional de Salud Pública; 2016 [fecha de publicación: 31 de octubre de 2016]. Disponible en: https://www.gob.mx/cms/uploads/attachment/ file/209093/ENSANUT.pdf.

2. Alhumah A, Gulam M, Khubeb M. Application of data mining: Diabetes health care in young and old patientes. Journal of King Saud UniversityComputer and Information Sciences. 2013;25(2):127-36.

3. Quinchia Castro A. Validez, correlación y concordancia entre calorimetría indirecta y el sistema de posicionamiento global, acelerometría triaxial y desglo- se de actividades para la determinación del gasto calórico en entrenamiento de resistencia en atletas de la modalidad 1. de Maestría. Colombia: Universidad Nacional de Colombia; 2018.

4. Adeyemo O, Adeyeye T. Comparative study of ID3/C4.5 Decision tree and multilayer perceptron algorithms for prediction of typhoid fever. African Journal of Computing \& ICT. 2015;8(1):103-12.

5. Blasco R. Gasto energético en reposo. Métodos de evaluación y aplicaciones. Rev Esp Nutr Comunitaria. 2015;21(1):243-51.

6. García Gutiérrez JA. Líneas de investigación en minería de datos en aplicaciones en ciencia e ingeniería. Estado del arte y perspectivas. España: Universidad Nacional de Educación a Distancia; 2016.

7. Meleksen A, Eyduran E, Reed BM. Use of RSM and CHAID data mining algorithm for predicting mineral nutrition of hazelnut. Plant Cell, Tissue and Organ Culture. 2017;128:303-16.

8. Obenshain M. Application of data mining techniques to healthcare. Infection Control and Hospital Epidemiology. 2004;25(8):690-5.

9. Shouman M, Turner T \& Stocker R.(2012). Using data mining techniques in heart disease diagnosis and treatment. Japan-Egypt Conference on electronics, communications and computers. [fecha de publicación: 12 de abril de 2012]. Disponible en: https://ieeexplore.ieee.org/xpl/conhome/6180280/proceeding.

10. VSrinivas K, Kavihta R, Govrdhan A. Application of data mining techniques in healthcare and prediction of heart attacks. Journal on Computer Science and Engineering. 2010;02(2):250-5.

11. Vargas M, Lancheros L, Barrera MP. Gasto energético en reposo y composición corporal en adultos. Rev Fac Med. 2011;59:43-58. 